\title{
Efficient Frequency Domain Forward Modeling of Elastic Waveforms
}

\author{
Ehsan Jamali Hondori, Hitoshi Mikada, Tada-nori Goto, and Junichi Takekawa
}

Dept. of Civil and Earth Res. Eng., Kyoto University

\begin{abstract}
We developed a Matlab package for finite difference frequency domain modeling of elastic waves in heterogeneous media which can be used to efficiently produce synthetic seismic data for full waveform inversion and modeling, or for wave propagation studies. By using 25-point finite difference stencil the number of necessary grid points per shortest shear wavelength reduced to 3.3 with an error smaller than $1 \%{ }^{1)}$. Although Matlab is a high level language, which is in general slower than other programing languages, the developed package exploits array processing ability of Matlab to compute the complex impedance matrix without including any loop in the algorithm. This brings a significant increase in computation performance and makes the package useful for developing realistic models. In order to suppress reflections from edges of the computation area Perfectly Matched Layers (PML) technique has been applied. Attenuation characteristics could be modeled easily by introducing complex valued velocities in frequency domain. Some examples show the performance of the package in modeling elastic waves.
\end{abstract}

\section{INTRODUCTION}

Frequency domain modeling of seismic waves has been used for several years by researchers, some of them used finite element method $^{2)}$ and some others used finite difference method ${ }^{3,4,5}$. It found to be more interesting for waveform studies because the attenuation behavior of the media could be considered by introducing complex valued elastic parameters. Also, monochromatic modeling at any desired frequency, parallel computing, and multisource processing are implemented easily. However, to get acceptable accuracy in frequency domain more grid points are required compared to time domain modeling. Pratt ${ }^{3,4)}$ applied the finite difference frequency domain method for crosshole tomography and seismic modeling with at least 15 grid points per shortest wavelength. Jo et al. ${ }^{6}$ proposed a 9-point finite difference operator including two different coordinate systems, one of them was standard system and the other one was rotated by 45 degrees, to solve the scalar wave equation. They could reduce the number of grid points to 5 per shortest wavelength with errors in results within $1 \%$. Shin and Sohn ${ }^{7)}$ introduced a 25-point operator for acoustic wave modeling which could reduce the number of grid points to 2.5 with errors less than $1 \%$.

In case of elastic wave modeling Stekl and Pratt ${ }^{8)}$ used the same strategy developed by Jo et al. ${ }^{6}$ and could reduce the number of necessary grid points per shortest shear wavelength to 4 for an error less than $5 \%$. Min et al. ${ }^{1)}$ proposed a finite difference scheme which is more accurate and reduces the number of grid points per shear wavelength to 3.3 for errors smaller than $1 \%$. They use 25-point finite difference computational stencil for 2D elastic wave equation. We used the method of Min et al. ${ }^{1)}$ to develop our elastic waveform forward modeling software. In order to truncate the computational area in the finite difference mesh we applied Perfectly Matched Layers (PML) in the frequency domain. Since the boundary conditions are applied directly in the frequency domain no axillary variable is needed for the PML application. In the following sections we will describe details of the finite difference operators and PML boundary conditions and show some results.

\section{THEORY}

\section{(1) Finite Difference Operators}

Here we present the method which Min et al. ${ }^{1)}$ used to solve the elastic wave equation. In the frequency domain, one can write the $2 \mathrm{D}$ elastic wave equation as bellow

$$
\begin{aligned}
& \omega^{2} \rho u+\frac{\partial}{\partial x}\left[\lambda\left(\frac{\partial u}{\partial x}+\frac{\partial v}{\partial z}\right)+2 \mu \frac{\partial u}{\partial x}\right] \\
& +\frac{\partial}{\partial z}\left[\mu\left(\frac{\partial v}{\partial x}+\frac{\partial u}{\partial z}\right)\right]+f_{x}=0
\end{aligned}
$$




$$
\begin{aligned}
& \omega^{2} \rho v+\frac{\partial}{\partial z}\left[\lambda\left(\frac{\partial u}{\partial x}+\frac{\partial v}{\partial z}\right)+2 \mu \frac{\partial v}{\partial z}\right] \\
& +\frac{\partial}{\partial x}\left[\mu\left(\frac{\partial v}{\partial x}+\frac{\partial u}{\partial z}\right)\right]+f_{z}=0
\end{aligned}
$$

where, $u, v, \omega, \rho, \lambda$, and $\mu$ are horizontal and vertical displacements in frequency domain, angular frequency, bulk density, and Lame parameters, respectively. $f_{x}$ and $f_{z}$ are horizontal and vertical external forces. We used 25-point finite difference stencil which was introduced by Min et al. ${ }^{1)}$ to discretize the wave equation. They defined as many finite difference operators as possible for spatial derivatives and then average them by weighting coefficients. For the mass acceleration terms they distributed the mass to all the 25 points by different coefficients. The homogeneous wave equation requires second order spatial derivatives which will be calculated by the stencils shown in Figure 1. For the $\partial^{2} / \partial x^{2}$ terms one can use the stencil in Figure 1a. In each row, five grid points can be used to form two centered finite difference operators. First operator is based on colored circles and the second one is based on unfilled circles. Then each operator is averaged by the appropriate coefficients, $c$ for colored circles and $d$ for unfilled circles. Resulted finite difference operators from each row will then be averaged by the coefficients $b_{1}, b_{2}$, and $b_{3}$ to give an accurate operator. For the $\partial^{2} / \partial z^{2}$ terms the similar procedure can be followed as shown in Figure 1b. Partial differentials of the form $\partial^{2} / \partial x \partial z$ can be discretized by using the finite difference stencil shown in Figure 1c. In this case two operators can be produced and averaged by coefficients $e$ and $f$. Figure 1d shows the distribution of weighting coefficients which have been used to average mass acceleration terms, $\rho \omega^{2} u$ and $\rho \omega^{2} v$. All the 25 points in the stencil contribute to form the mass acceleration. The formulation for the heterogeneous case is similar and the method of Kelly et al. ${ }^{9)}$ can be used. Since the finite differenced equations and weighting coefficients are available in Min et al. ${ }^{1)}$ we move to the next section which describes PML application.

\section{(2) Perfectly Matched Layers Boundary Conditions}

Numerical modeling of wave equations from an unbounded media requires truncation of the computational area because of the limitations in memory and computation time. As a consequence, reflections from truncated boundaries will appear in

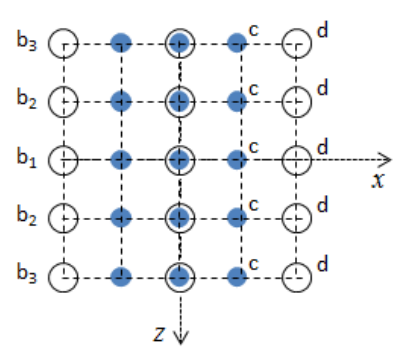

(a)

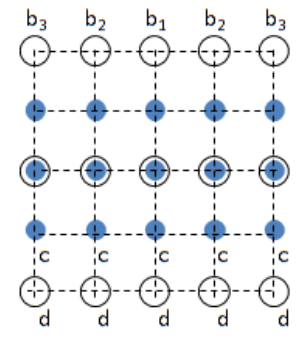

(b)

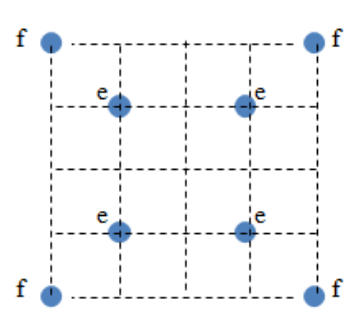

(c)

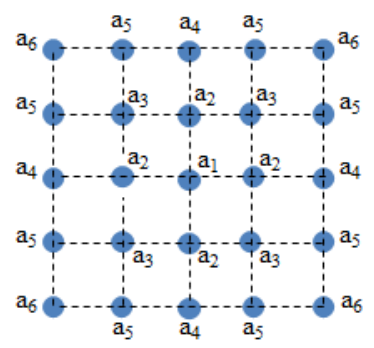

(d)

Figure 125 -point finite difference stencils for spatial derivatives and mass acceleration terms.

the solutions of the wave equation. In order to achieve accurate results one needs to suppress any reflection from the boundaries as much as possible. Various methodologies have been developed for this purpose including paraxial approximation of wave equation ${ }^{10)}$, sponge damping of reflections ${ }^{11)}$, perfectly matched layers (PML) ${ }^{12)}$, etc. Among all the methods for boundary conditions PML, which was first developed for the electromagnetic waves by Berenger ${ }^{13)}$, is found to be the most effective one to suppress boundary reflections. Here we use the PML in frequency domain which is efficient and easy to apply. To include PML boundary conditions in the elastic wave equation we need to transform the wave equation to the complex coordinate system and define complex stretching functions as $^{14)}$

$$
\varepsilon_{x}=1+\frac{\sigma_{x}}{i \omega} \quad, \quad \varepsilon_{z}=1+\frac{\sigma_{z}}{i \omega}
$$

where the attenuation function $\sigma$ represents the damping behavior of the PML zone. The spatial derivatives in the stretched coordinate PML area are represented as bellow

$$
\frac{\partial}{\partial \tau} \cdot 1 \frac{\partial}{\tau}, \quad \frac{\partial}{\partial \tau} \cdot 1 \frac{1}{\tau} .
$$

Using equation (3) one can easily derive the frequency domain wave equation for the PML area. Since there is no need to transform the equations to the time domain, no axillary variable is needed and the required memory will not increase significantly. 


\section{(3) Modeling Attenuation of Seismic Waves}

Attenuation behavior of the media can be considered by including complex valued velocities in the frequency domain. We used the method of Kolsky-Futterman ${ }^{14)}$ which has been applied in a wide range of geophysical modeling. The complex velocity could be calculated as bellow

$$
\frac{1}{c(\omega)}=\frac{1}{c}+\frac{1}{\pi c Q} \ln \left|\frac{\omega_{r}}{\omega}\right|+i \frac{\operatorname{sgn}(\omega)}{2 c Q}
$$

where, $c, \omega, \omega_{r}$, and $Q$ are the velocity, angular frequency, reference frequency, and attenuation factor, respectively.

\section{RESULTS}

Figure 2 shows wave propagation snapshots in a homogeneous media with $\mathrm{V}_{\mathrm{p}}=3000 \mathrm{~m} / \mathrm{s}, \mathrm{V}_{\mathrm{s}}=1500$ $\mathrm{m} / \mathrm{s}$, and $\rho=2200 \mathrm{Kg} / \mathrm{m}^{3}$. This figure shows that PML boundary condition could efficiently suppress the artificial reflections from the edges of the computation area. Explosive source with dominant frequency of $5 \mathrm{~Hz}$ is located at the center of the model and maximum modeled frequency is $30 \mathrm{~Hz}$. Grid size is $150 \times 150$ with an interval of $10 \mathrm{~m}$. Top left and right panels show the snapshots at times 350 and $583 \mathrm{~ms}$, respectively. Bottom left and right panels show vertical and horizontal displacements, respectively. As Figure 2 shows, no reflections from the boundaries are produced.
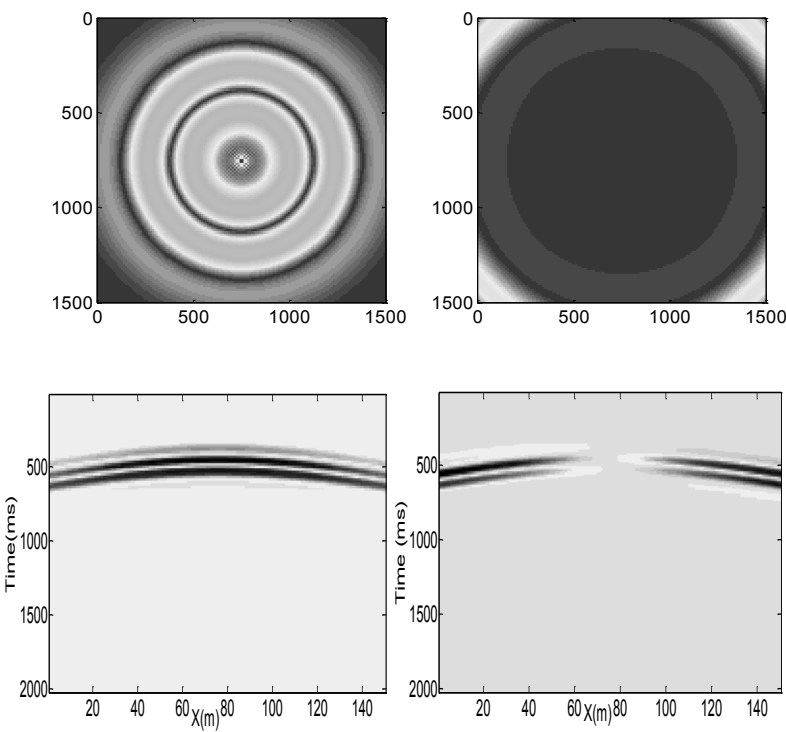

Figure 2 top: snapshots at $350 \mathrm{~ms}$ (left) and $583 \mathrm{~ms}$ (right) from wave propagation in homogeneous media, bottom: vertical (left) and horizontal (right) displacements.
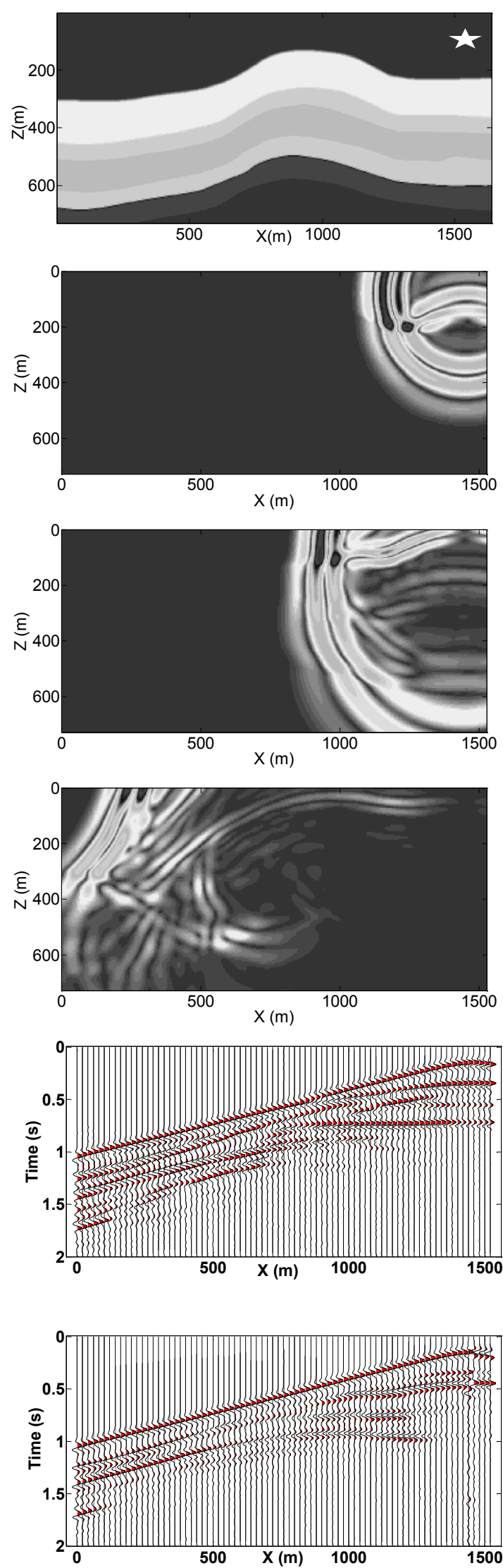

Figure 3 Top to down: geological model, snapshots of wave propagation at times 290, 460, and $890 \mathrm{~ms}$, vertical and horizontal displacements. 

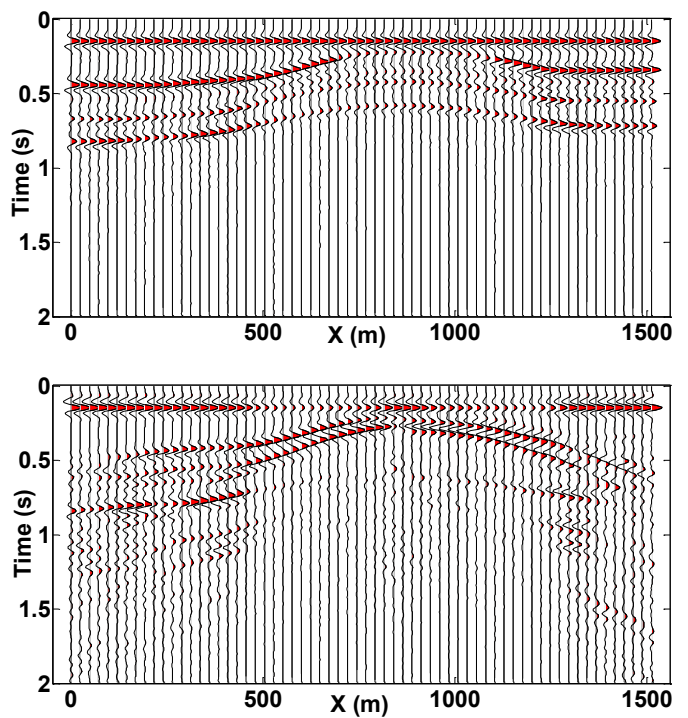

Figure 4 Zero offset sections of vertical (top) and horizontal (bottom) displacements.

Second example represents elastic wave propagation in a layered medium including an anticline shape. Figure 3 shows the geological feature, wave propagation snapshots, and recorded seismic traces of the model. Explosive seismic source with dominant frequency of $10 \mathrm{~Hz}$ is located at a depth of $80 \mathrm{~m}$ near to top right corner of the media (the starred point in Figure 3). Receivers are located at the surface and seismograms for 2 seconds have been recorded. Shear and compressional wave velocities range between $900-1900 \mathrm{~m} / \mathrm{s}$ and $1550-3200 \mathrm{~m} / \mathrm{s}$, respectively. Maximum modeled frequency is $50 \mathrm{~Hz}$. Model size is $728 \mathrm{~m} \times 1528 \mathrm{~m}$ and grid point interval is $4 \mathrm{~m}$. Figure 4 shows zero offset sections of the vertical and horizontal displacements. The figure clearly illustrates the geological features of the model. The horizontal events on top of the zero offset sections are first arrivals.

\section{CONCLUSIONS}

A package for finite difference frequency domain modeling of the elastic waveforms has been successfully developed. Exploiting 25-point stencils the number of required grid points per shortest shear wavelength could be reduced to 3.3. By using array processing algorithms in Matlab, complex impedance matrix with high ranks could be calculated very efficiently. Perfectly matched layers could suppress artificial boundary reflections and attenuation behavior of the media has been considered by using complex valued velocities. According to the results, the developed software can be used in forward simulations of full waveform inversion and modeling problems.

\section{REFERENCES}

1) Min, D., Shin, C., Kwon, B., and Chung, S., 2000, Improved frequency-domain elastic wave modeling using weighted-averaging difference operators: Geophysics, 65, 3, 884-895.

2) Marfurt, K. J., 1984, Accuracy of finite-difference and finite-element modeling of the scalar and elastic wave equations: Geophysics, 49, 533-549.

3) Pratt, R. G., 1990a, Inverse theory applied to multi-source crosshole tomography, part II: Elastic wave-equation method: Geophys. Prosp., 38, 287-310.

4) Pratt, R. G., 1990b, Frequency-domain elastic wave modeling by finite differences: A tool for crosshole seismic imaging: Geophysics, 55, 626-632.

5) Amini, N., Javaherian, A., 2011, A MATLAB-based frequency-domain finite difference package for solving 2D visco-acoustic wave equation: Waves in Random and Complex Media, 21: 1, 161-183.

6) Jo, C. H., Shin, C., and Suh, J. H., 1996, An optimal 9-point, finite difference, frequency space, 2-D scalar wave extrapolator: Geophysics, 61, 529-537.

7) Shin, C., and Sohn, H., 1998, A frequency-space 2-D scalar wave extrapolator using extended 25-point finite-difference operators: Geophysics, 63, 289-296.

8) Stekl, I., and Pratt, R. G., 1998, Accurate viscoelastic modeling by frequency-domain finite differences using rotated operators: Geophysics, 63, 1779-1794.

9) Kelly, K. R., Ward, R. W., Treitel, S., and Alford, R. M., 1976, Synthetic seismograms: A finite-difference approach: Geophysics, 41, 2-27.

10) Clayton, R., and Enquist, B., 1977, Absorbing boundary conditions for acoustic and elastic wave equations: Bull. Seis. Soc. Am., 67, 1529-1540.

11) Shin C., 1995, Sponge boundary condition for frequency-domain modeling: Geophysics, 60, $1870-1874$

12) Drossaert, F., H., and Giannopoulos, A., 2007, A non-split complex frequency-shifted PML based on recursive integration for FDTD modeling of elastic waves: Geophysics, 72, 2, T9-T17.

13) Bérenger, J. P., 1994, A perfectly matched layer for absorption of electromagnetic waves: Journal of Computational Physics, 114, 185-200.

14) Futterman, W., I., 1962, Dispersive body waves: Journal of Geophysical Research, 67, 5279-5291. 